\title{
Recent Advances in the Chemistry of $\mathrm{Sml}_{2}-\mathrm{H}_{2} \mathrm{O}$
}

\author{
Brice Sautier and David J. Procter
}

\begin{abstract}
Recent work from our laboratories has shown $\mathrm{Sml}-\mathrm{H}_{2} \mathrm{O}$ to be a versatile, readily-accessible and nontoxic reductant that is more powerful than $\mathrm{Sml}_{2}$. This review describes the reduction of functional groups that were previously thought to lie beyond the reach of $\mathrm{Sml}_{2}$ and complexity-generating cyclisations and cyclisation cascades triggered by the reduction of the ester carbonyl group with $\mathrm{Sml}_{2}-\mathrm{H}_{2} \mathrm{O}$.
\end{abstract}

Keywords: Cyclisations $\cdot$ Radicals $\cdot$ Reductions $\cdot$ Samarium $\cdot$ Water

\section{Introduction}

First introduced in 1977 by Kagan, ${ }^{[1]}$ samarium diiodide ( $\mathrm{SmI}_{2}$, Kagan's reagent) is a versatile single-electron reducing agent. The reagent is able to mediate a wide range of radical and anionic transformations, often with high regio-, stereoand chemoselectivity, and has thus been used widely in critical transformations on sensitive substrates, for example in natural product syntheses, ${ }^{[2-5]}$ and to generate high complexity in cascade reactions. A major advantage of $\mathrm{SmI}_{2}$ arises from the ability to tune its reducing power using additives and co-solvents (e.g. (vs. $\mathrm{Ag} / \mathrm{AgNO}_{3}$ ) $\mathrm{SmI}_{2}$ : $-1.33 \mathrm{~V} ; \mathrm{SmI}_{2}-\mathrm{H}_{2} \mathrm{O}:-1.9 \mathrm{~V} ; \mathrm{SmI}_{2}-\mathrm{HMPA}$ : $-2.05 \mathrm{~V}){ }^{[6]}$ This has led to the discovery of new Sm(II)-based reagent systems that have opened up unexplored reaction space through the manipulation of new substrates. In recent years, our group has shown that $\mathrm{SmI}_{2}-\mathrm{H}_{2} \mathrm{O}$ can mediate a range of new reactions involving functional groups and substrates that were previously thought to lie beyond the reach of $\mathrm{SmI}_{2}$. In this review, we present an overview of recent work from our laboratories employing $\mathrm{SmI}_{2}-\mathrm{H}_{2} \mathrm{O}$, starting with the discovery of new functional group reductions and concluding with their application in cyclisations and cyclisation cascades.

\footnotetext{
${ }^{\star}$ Correspondence: Prof. D. J. Procter

University of Manchester

School of Chemistry

Oxford Road

Manchester M13 9PL

Tel.: +44 1612751425

Fax: +44 1612754939

E-mail: david.j.procter@manchester.ac.uk
}

\section{Discovery of New Reactivities}

The potential of combining $\mathrm{SmI}_{2}$ and $\mathrm{H}_{2} \mathrm{O}$ was recognised during the pioneering studies of Kagan, ${ }^{[1]}$ and the accelerating effect of $\mathrm{H}_{2} \mathrm{O}$ on the reactions of $\mathrm{SmI}_{2}$ with less-reactive functional groups was noted by Curran in 1993. ${ }^{[7]}$ However, only in recent studies by Flowers has it been shown that the use of excess $\mathrm{H}_{2} \mathrm{O}$ results in the formation of a stronger $\mathrm{Sm}$ (II) reductant. ${ }^{[8]}$ Flowers' results indicate that water exhibits a much higher affinity for $\mathrm{SmI}_{2}$ than other proton sources, displacing the bulk solvent from the inner coordination sphere even at low concentration (50 equiv. with respect to $\mathrm{SmI}_{2}$ ) and resulting in a change of mechanism for the reduction of acetophenone at higher water concentration (>80 equiv.). ${ }^{[9]}$ Cyclic voltammetry studies determined a reduction potential of $-1.9 \mathrm{~V}$ for $\mathrm{SmI}_{2}$ in the presence of 500 equiv. of water ( $v s . \mathrm{Ag}$ / $\mathrm{AgNO}_{3}$ ). In 2009, Hoz reported that the use of water with SmI results in rapid trapping of radical anions by protonation from water bound to the samarium centre with proton transfer from the bulk solution being insignificant. ${ }^{[10]}$ More recently, Flowers has demonstrated that water displaces the iodides from the inner coordination sphere of samarium. ${ }^{[11]}$ In addition, coordinatively saturated complexes were not formed even at high water concentration, and thus substrate binding to samarium and reduction was not inhibited. The exciting properties of $\mathrm{SmI}_{2}-\mathrm{H}_{2} \mathrm{O}$ have been exploited by our group to uncover new reactivity and selectivity in the chemistry of important functional groups.

\subsection{Selective Lactone Reduction}

During studies on the development of stereoselective $\mathrm{SmI}_{2}$-mediated conjugate reduction/spirocyclisations, we found that treatment of 1 with $\mathrm{SmI}_{2}$ in THF using $\mathrm{H}_{2} \mathrm{O}$ as a co-solvent gave a mixture of the expected lactone $\mathbf{2}$ and triol $\mathbf{3}$ (Scheme
1). ${ }^{[12,13]}$ Prior to this observation, the reduction of unactivated aliphatic esters or lactones with $\mathrm{SmI}_{2}$ was thought to be impossible.

Importantly, the presence of a $\beta$-hydroxyl was found not to be crucial and the scope of the reaction was explored using less-functionalised lactones. In all cases, six-membered lactones underwent reduction to the corresponding diols in good yield. The reduction of lactones bearing acyclic ester substituents was also investigated, and the corresponding diols were obtained in excellent yields with no reduction of the acyclic ester moiety (Scheme 2). [13,14]

The selectivity of the $\mathrm{SmI}_{2}-\mathrm{H}_{2} \mathrm{O}$ reagent system was emphasised using competition experiments in which mixtures of six-membered lactones and esters, or other lactones, were prepared and treated with $\mathrm{SmI}_{2}-\mathrm{H}_{2} \mathrm{O}$. In all cases, selective reduction of the six-membered lactone occurred smoothly and no products arising from reduction of other lactones and acyclic esters were observed (Scheme 3).

Experimental and theoretical studies were carried out to explore the mechanism and selectivity of the reduction. ${ }^{[14]}$ Reductions using $\mathrm{SmI}_{2}-\mathrm{D}_{2} \mathrm{O}$ delivered labelled products consistent with a mechanism involving the generation and protonation of anions during a series of single electron transfers (Scheme 4). Studies suggested that the ring-size selectivity of the transformation arises from the initial electron transfer to the lactone carbon-

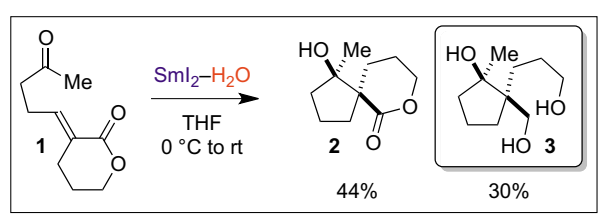

Scheme 1. The observation of lactone reduction during studies on stereoselective spirocyclisation. 


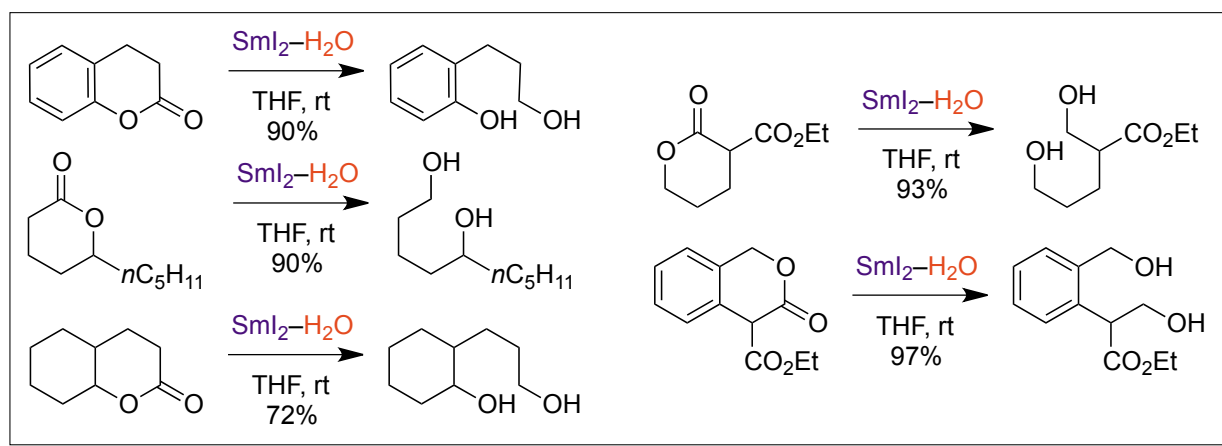

Scheme 2. The reduction of six-membered lactones.

yl. The ketyl radical-anion arising from electron transfer to the carbonyl of a sixmembered lactone benefits from stabilisation by an anomeric effect and it is this stabilisation that appears to promote the initial reduction step. Calculations on the lowest energy conformation of the radical anion derived from a six-membered lactone support a pseudoaxial, anomerically stabilised orientation. Interestingly, 2-oxabicyclo[2.2.2] octan-3-one, a conformationally-locked six-membered lactone that cannot adopt the chair conformation necessary for effective anomeric stabilisation of an intermediate radical-anion, was not reduced by $\mathrm{SmI}_{2}-\mathrm{H}_{2} \mathrm{O}$. In addition, computational studies on the relative reaction energy of the initial electron-transfer to the lactone carbonyl gave a calculated energy considerably lower for a six-membered lactone than for analogous five- and sevenmembered lactones. The calculated energy of the second electron transfer was similar for all three systems. This later observation agreed with kinetic studies that suggested the first electron transfer is the rate determining step (Scheme 4).

\subsection{Selective 1,3-Diester Reduction}

Cyclic 1,3-diesters (e.g. Meldrum's acids) are also selectively reduced using $\mathrm{SmI}_{2}-\mathrm{H}_{2} \mathrm{O}$ to afford the corresponding 3-hydroxy propanoic acids (Scheme 5). ${ }^{[15]}$ Meldrum's acids are important chemical feedstocks for synthesis and ours is the first example of the desymmetrising, mono-reduction of such a system. Prior to our report, access to hydroxy acids from the corresponding Meldrum's acids typically required four steps (e.g. conversion to the monoacid, activation of the acid as a mixed anhydride, reduction using $\mathrm{NaBH}_{4}$ and hydrolysis).

Competition experiments carried out on mixtures of cyclic 1,3-diesters and acyclic 1,3-diesters illustrated the impressive selectivity of the reagent system (Scheme 6).

A mechanism analogous to that proposed for the reduction of six-membered lactones was suggested: Initial electron

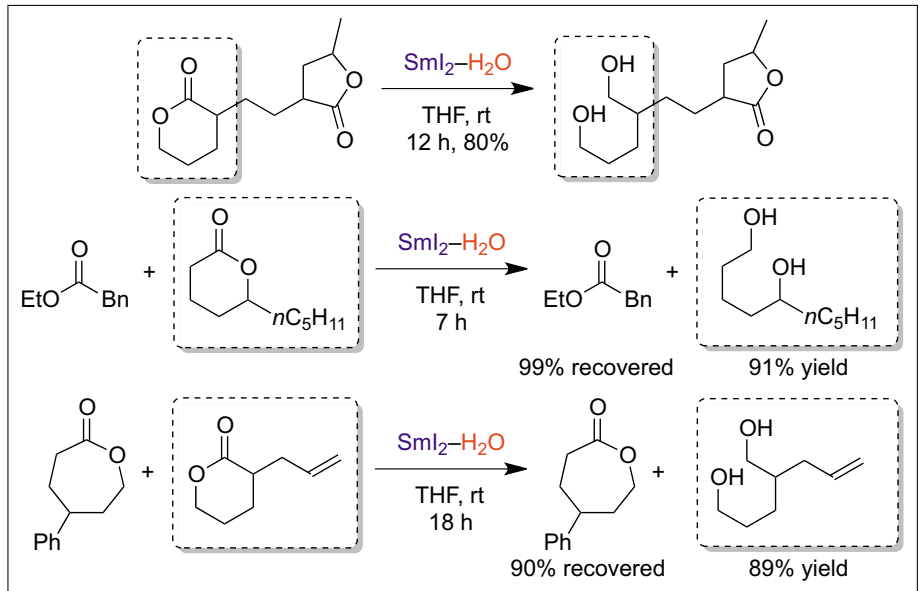

Scheme 3. Competition experiments illustrating the selectivity of $\mathrm{Sml}_{2}-\mathrm{H}_{2} \mathrm{O}$.

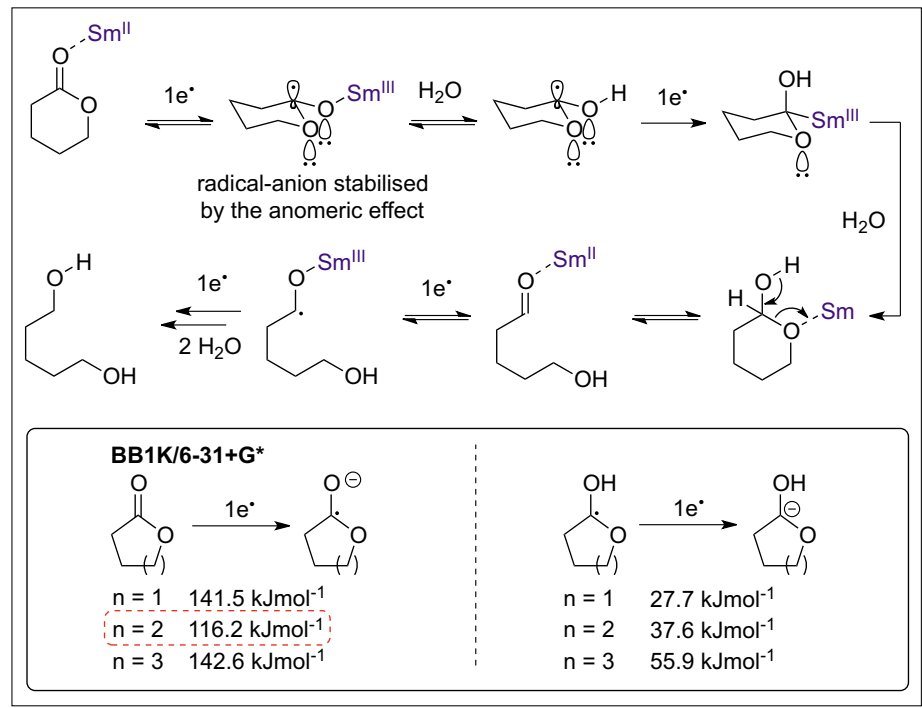

Scheme 4. Proposed mechanism of lactone reduction and the origin of selectivity.

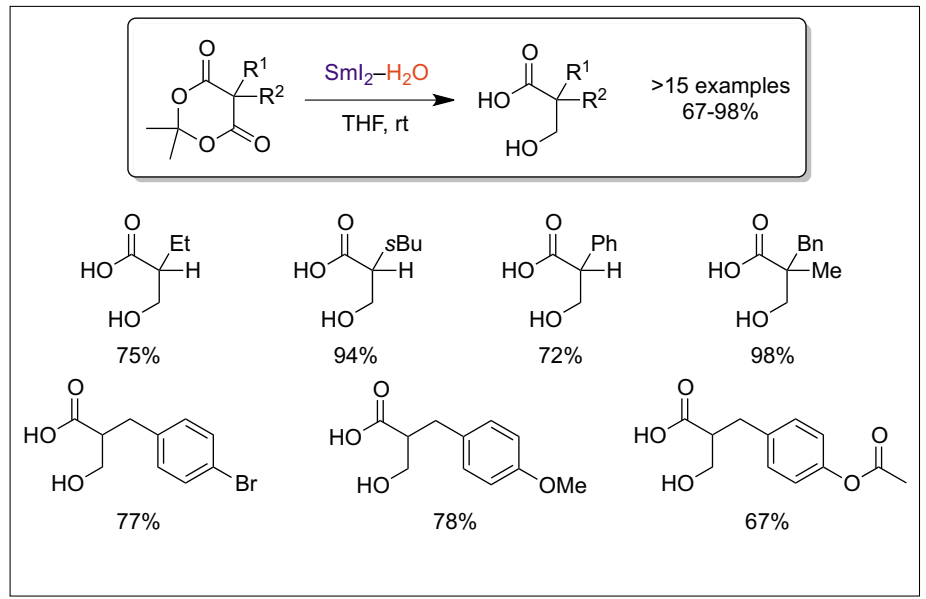

Scheme 5. Selective reduction of Meldrum's acid derivatives.

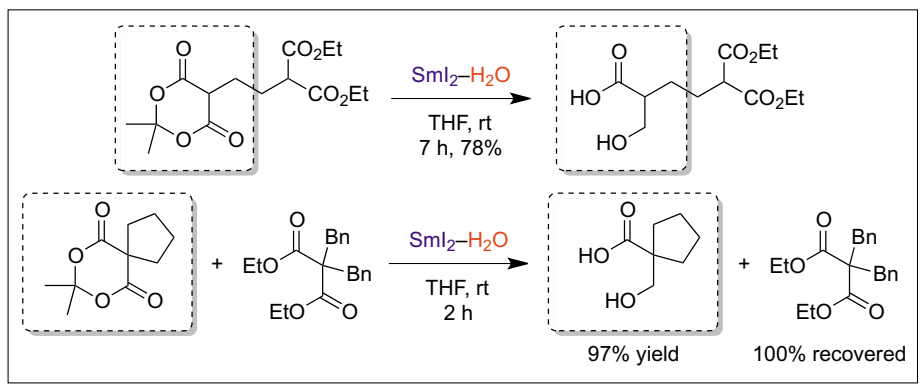

Scheme 6. Competition experiments illustrating the selectivity of $\mathrm{Sml}_{2}-$ $\mathrm{H}_{2} \mathrm{O}$. 


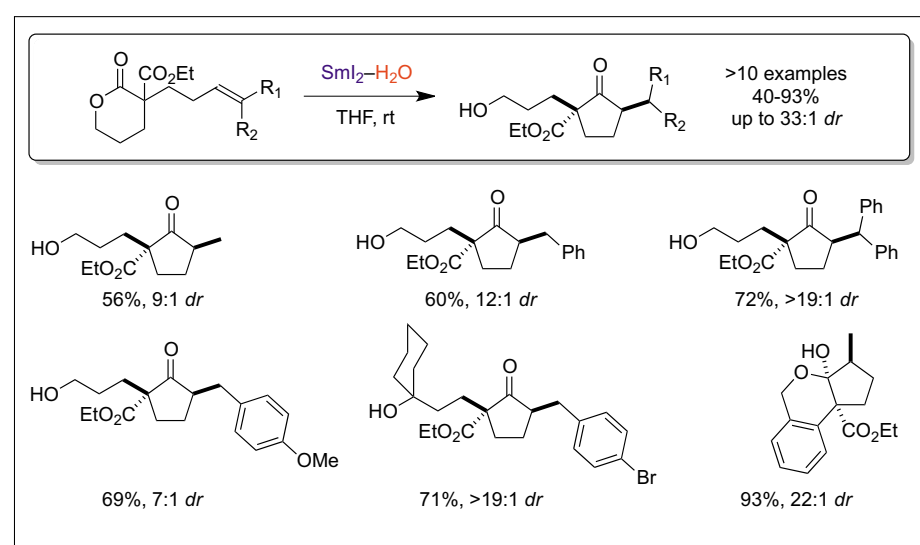

Scheme 7. The cyclisation of unsaturated lactones to give cyclopentanones.

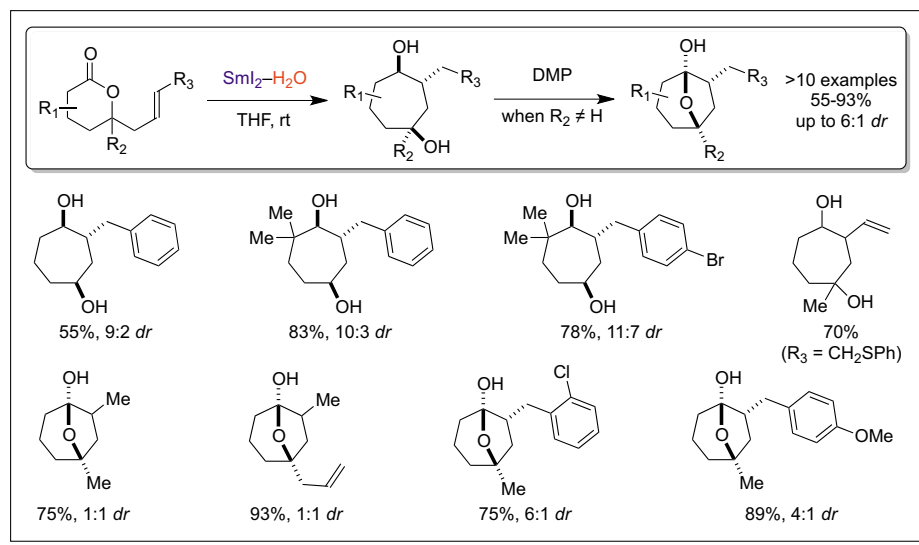

Scheme 8. The cyclisation of unsaturated lactones to give cycloheptanediols. transfer from $\mathrm{Sm}(\mathrm{II})$ to the ester carbonyl forms a pseudoaxial ketyl radicalanion stabilised by an anomeric effect. Subsequent reduction of the ketyl radicalanion to the anion, protonation, collapse of the ketal unit and reduction of the resulting aldehyde then affords the hydroxy acid product. Further reduction to the diol is prevented by collapse of the cyclic system. Calculations show a sizeable difference in relative reaction energy for electrontransfer to the ester carbonyl of cyclic and acyclic 1,3-diesters, with the former being lower in energy. ${ }^{[14]}$

\section{Exploiting the Radical-anion Intermediates in C-C Bond Formation}

\subsection{Cyclisations of Lactones}

Upon treatment with $\mathrm{SmI}_{2}-\mathrm{H}_{2} \mathrm{O}$, sixmembered lactones bearing a tethered alkene at the $\alpha$-position afforded cyclopentanones in good yields and with high diastereoselectivity through an unprecedented radical cyclisation (Scheme 7). ${ }^{15]}$ The presence of an $\alpha$-ester appears to prevent the collapse of a hemiketal intermediate and over-reduction to yield cyclopentanol products. These cyclisations demonstrated for the first time the feasibility of using an ester carbonyl as a ketyl radical precursor in carbonyl-alkene couplings.

In addition, tethering the alkene at the $\delta$ position of the six-membered lactone gave access to seven-membered carbocycles in moderate to good yield (Scheme 8). ${ }^{[16]}$ In contrast to the examples shown in Scheme 7 , ketone intermediates were further reduced to give cyclic alcohol products under the $\mathrm{SmI}_{2}-\mathrm{H}_{2} \mathrm{O}$ conditions.

\subsection{Cyclisations of Cyclic 1,3-Diesters}

Analogous cyclisations of cyclic 1,3-diesters bearing tethered alkenes were also explored.[15a,17] Cyclopentanols were obtained in good yields from Meldrum's

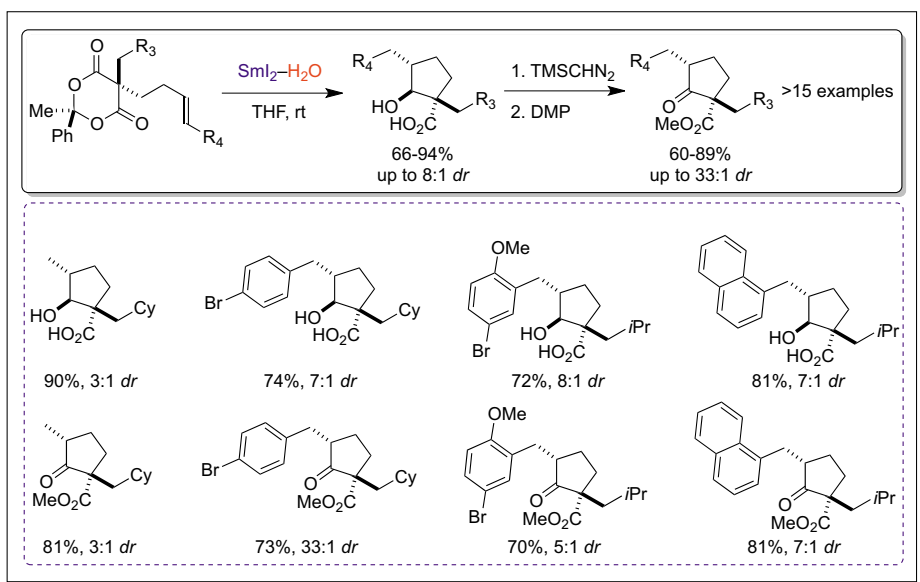

Scheme 9. The cyclisation of unsaturated cyclic 1,3-diesters to give cyclopentanols. acid-derived substrates, although diastereoselectivities were only moderate. Improved diastereoselectivites were observed when the acetone-derived ketal was exchanged for an acetophenone-derived ketal. The improved selectivity appears to arise from the greater conformational control exerted by the acetophenone ketal unit over the substrate and the intermediate radical anion.

Surprisingly, studies on the influence of the reaction temperature showed that higher selectivities could be obtained by increasing the reaction temperature from ambient to $50{ }^{\circ} \mathrm{C}$. Again, ketone intermediates were reduced to cyclopentanol products under the reaction conditions (Scheme 9).

\section{Radical Cyclisation Cascades}

\subsection{Cyclisation Cascades of Lactones}

We next considered the possibility of trapping both ketyl-radical anions formed during the reduction of the ester carbonyl. We proposed that cis-lactones bearing two tethered alkenes, such as $\mathbf{5}$, would undergo selective cascade cyclisations. ${ }^{[16]}$ Theoretical calculations on model substrate 5 suggested that high sequence integrity should be observed due to the low relative activation energy calculated for the cyclisation of axial radical-anion 4 onto the 'right' alkene (Scheme 10).

Several cis-configured substrates were synthesised and treated with $\mathrm{SmI}_{2}-\mathrm{H}_{2} \mathrm{O}$. Pleasingly, the expected azulenes were obtained in good to excellent yields and with good diastereoselectivity (Scheme 11). The successful use of alkyne tethers in the second stage of the cascade allowed the installation of an exocyclic olefin that could be used for further functionalisation. The importance of the cis-configuration in the substrates was confirmed when a transconfigured lactone substrate was found to undergo monocyclisation onto the 'left' alkene to give a cyclopentanol upon exposure to $\mathrm{SmI}_{2}-\mathrm{H}_{2} \mathrm{O}$.

\subsection{Cyclisation Cascades of Meldrum's Acids}

The potential of cyclic 1,3-diesters bearing two tethered alkenes to undergo analogous cyclisation cascades was also investigated. The desymmetrisation of readily accessible cyclic 1,3-diester derivatives was achieved, affording cis-fused bicyclic hydroxy acids in good yield and with high diastereocontrol.[17,18] The use of substrates bearing different alkene substituents led to single isolated diastereoisomers in moderate to good yields (Scheme 12). ${ }^{[18]}$ Differential activation of the radical acceptors was found to be crucial in order 
to achieve high sequence integrity and thus high diastereocontrol. In addition, the use of different tether lengths was possible and alkynes could also be employed as the second radical acceptor.

In all cases, the stereochemistry obtained is consistent with the reaction of both radicals through anti-transition states (Scheme 13).

\subsection{Cyclisation Cascades Terminated by Lactone Reduction}

We have also exploited $\mathrm{SmI}_{2}-\mathrm{H}_{2} \mathrm{O}$ in cascade cyclisations terminated by lactone

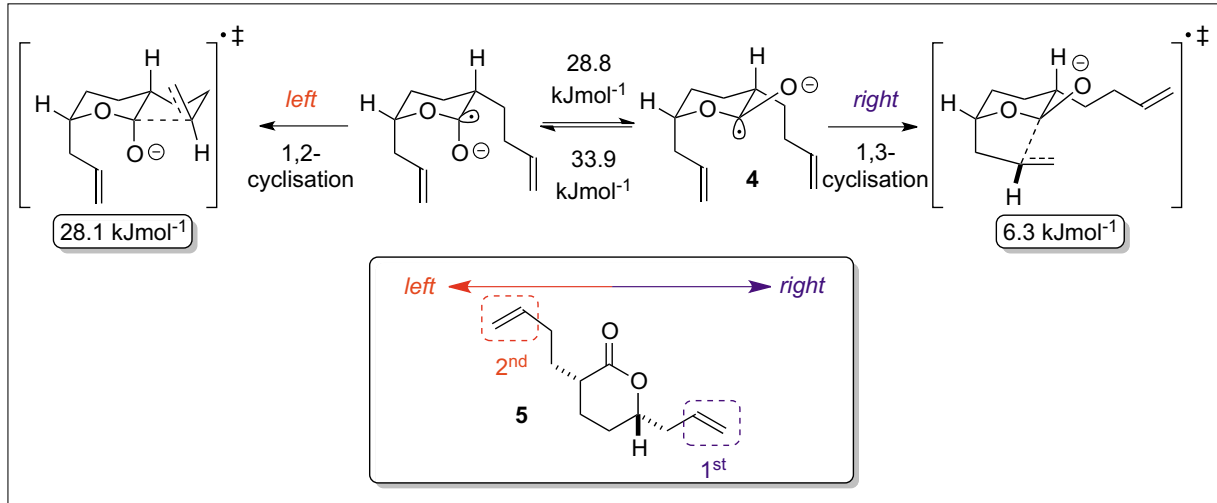

Scheme 10. Calculations to assess the feasibility of lactone cyclisation cascades.
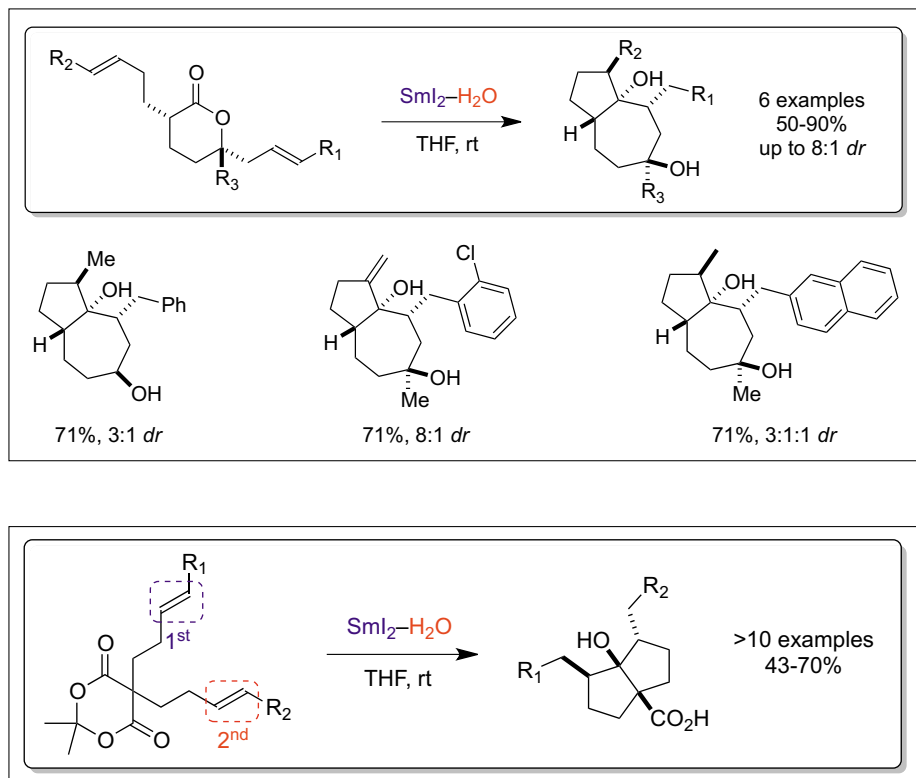

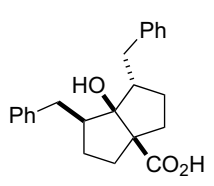

$70 \%$

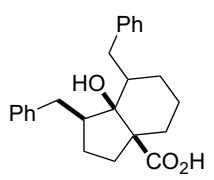

$67 \%, 1: 1.3 d r$

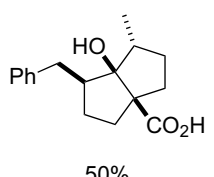

$50 \%$

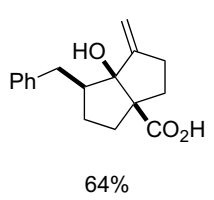

Scheme 11. Lactone radical cyclisation cascades.

Scheme 12.

Desymmetrising cyclisation cascades of unsaturated Meldrum's acid derivatives.

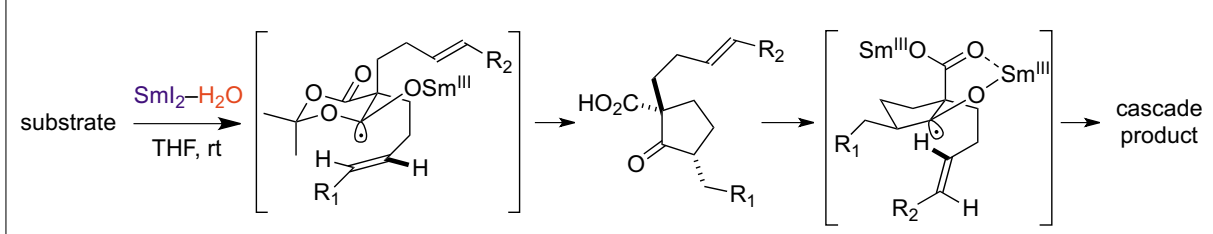

Scheme 13. Proposed origin of diastereoselectivity in the cyclisation cascades of Meldrum's acid derivatives. reduction. In synthetic studies towards stolonidiol, a natural cytotoxic diterpenoid, the reagent system was used for the conversion of enantiomerically pure lactone 6 to triol 7, in a single cascade process, in good yield and high stereocontrol.[19] The cascade starts with the conjugate reduction of the electron deficient olefin and formation of a Sm(III)-enolate which then undergoes a diastereoselective aldol cyclisation. The observed stereoselectivity can be explained by complexation of the acetate and ketone carbonyl groups with Sm(III) in the transition state. Subsequent reduction of the spirocyclic lactone intermediate then affords the desired triol (Scheme 14).

The $\mathrm{SmI}_{2}-\mathrm{H}_{2} \mathrm{O}$ reagent system also proved to be highly effective in a one-pot dialdehyde cyclisation cascade/lactone reduction sequence. ${ }^{[20]}$ Treatment of dialdehyde 8 with $\mathrm{SmI}_{2}-t \mathrm{BuOH}$ triggers an anti-selective ketyl-olefin cyclisation and selective formation of a $\mathrm{Sm}$ (III)-enolate which undergoes subsequent selective aldol cyclisation. Addition of $\mathrm{SmI}_{2}-\mathrm{H}_{2} \mathrm{O}$ to the reaction flask then triggers reduction of the six-membered lactone in the spirocyclic product, thus giving complex bicyclic tetraol 9 in excellent yield and diastereoselectivity (Scheme 15).

\section{Reduction of Acyclic Carboxylic Acid Derivatives Using $\mathrm{Sml}_{2}-\mathrm{H}_{2} \mathrm{O}$ Activated by Amine}

More recently, we have reported the use of $\mathrm{SmI}_{2}-\mathrm{H}_{2} \mathrm{O}$ in conjunction with an amine for the reduction of acyclic esters (Scheme 16). ${ }^{[21]}$ Crucially, this is the first example of the reduction of acyclic esters using a $\mathrm{SmI}_{2}$-based system. The reagent system takes advantage of the Lewis basicity of the amine to generate a stronger reductant. Importantly, both water and amine additives are required for ester reduction although the identity of the amine can be varied. Our studies show that a broad range of ester groups can be reduced, including sterically demanding substrates, leading to the corresponding alcohols in excellent

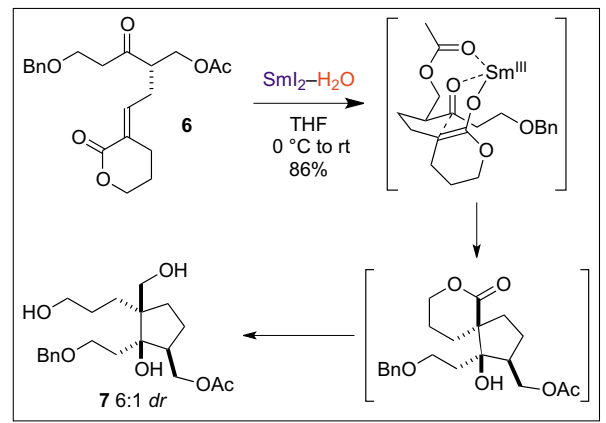

Scheme 14. A conjugate reduction/aldol spirocyclisation/lactone reduction cascade in studies towards stolonidiol. 


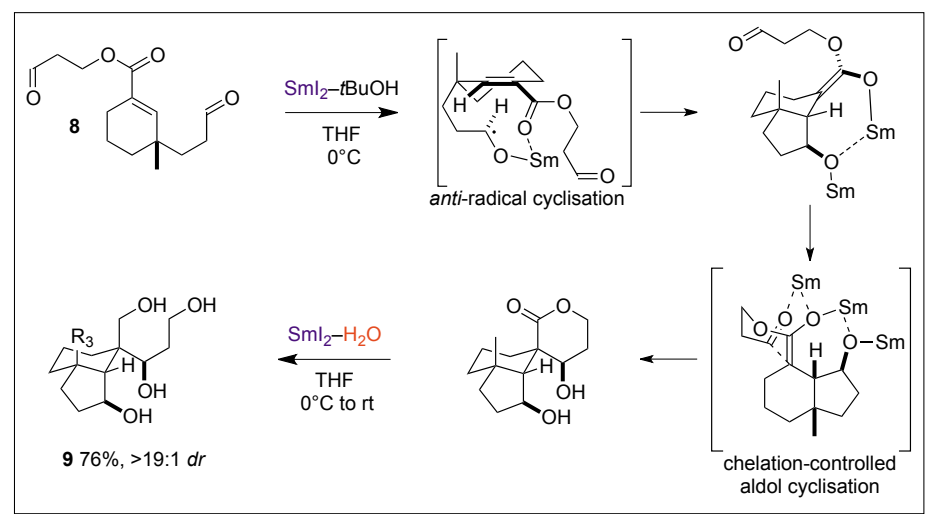

Scheme 15. A one-pot dialdehyde cyclisation cascade and lactone reduction.

yields. The reduction of lactones to the corresponding diols, irrespective of their ring size, can now be readily achieved using $\mathrm{SmI}_{2}-\mathrm{H}_{2} \mathrm{O}-\mathrm{Et}_{3} \mathrm{~N}$. ${ }^{[22]}$ As expected, selectivity for the reduction of lactones over that of acyclic esters can be achieved, as emphasised by the selective reduction of the lactone moiety in lovastatin, and the reagent system shows good tolerance of functional groups and steric hindrance. The $\mathrm{SmI}_{2}-$ $\mathrm{H}_{2} \mathrm{O}-\mathrm{Et}_{3} \mathrm{~N}$ reagent system is also able to reduce carboxylic acids to the corresponding alcohols, allowing us to disclose the first general protocol for their reduction under electron transfer conditions.[23] Again, good tolerance of functional groups and steric hindrance was observed. We believe that the mechanism of reduction with $\mathrm{SmI}_{2}-\mathrm{H}_{2} \mathrm{O}-\mathrm{Et}_{3} \mathrm{~N}$ mirrors that proposed for the reduction of lactones and involves a series of single electron transfer and protonation steps.

\section{Conclusion}

The emergence of $\mathrm{SmI}_{2}-\mathrm{H}_{2} \mathrm{O}$ as a readily-accessible, non-toxic, and more powerful reductant than $\mathrm{SmI}_{2}$ has led to the discovery of synthetically useful reactions that exhibit exquisite selectivity. The new carbonyl reductions developed within our group allow us to manipulate substrates that were previously thought to be inert to $\mathrm{SmI}_{2}$. Such reductions provide convenient access to 'unusual' radical anions that can be exploited in unprecedented $\mathrm{C}-\mathrm{C}$ bondforming events and have thus paved the way to new cyclisations and cyclisation cascades. Further studies will undoubtedly lead to a wealth of new transformations and selectivity that can not be achieved using conventional reagents thus making $\mathrm{SmI}-\mathrm{H}_{2} \mathrm{O}$ an indispensable tool for advances in synthetic chemistry.

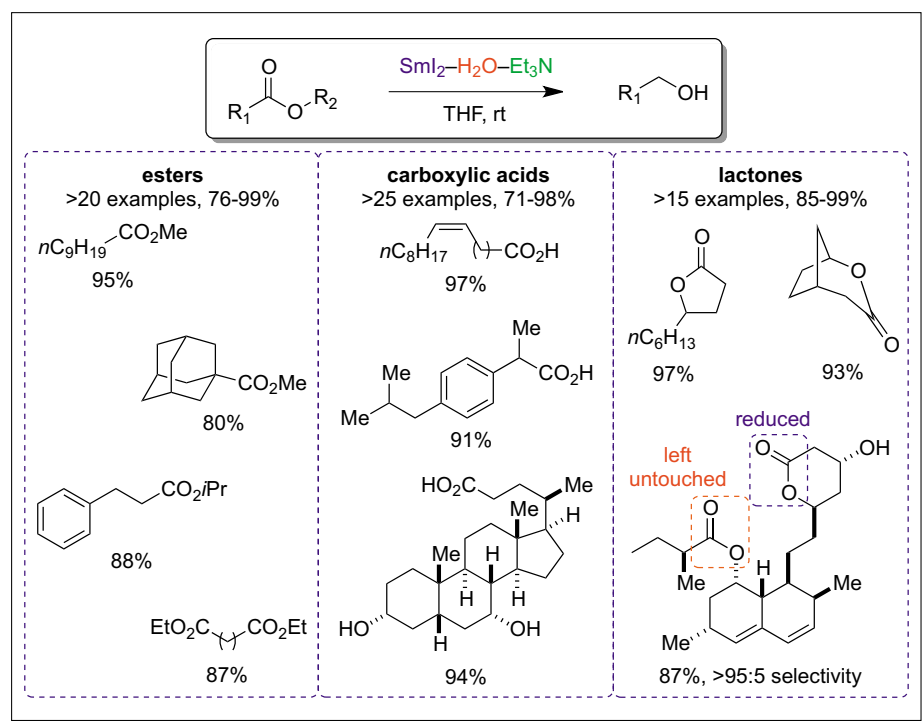

Scheme 16. The reduction of carboxylic acid derivatives using $\mathrm{Sml}_{2}-$ $\mathrm{H}_{2} \mathrm{O}-\mathrm{Et}_{3} \mathrm{~N}$.
[1] a) J. L. Namy, P. Girard, H. B. Kagan, Nouv. J. Chim. 1977, 1, 5; b) P. Girard, J. L. Namy, H. B. Kagan, J. Am. Chem. Soc. 1980, 102, 2693.

[2] D. J. Procter, R. A. Flowers, II, T. Skrydstrup, 'Organic Synthesis using Samarium Diiodide: A Practical Guide', RSC Publishing, Cambridge, 2009.

[3] For general reviews on $\mathrm{SmI}_{2}$, see: a) $\mathrm{H}$. B. Kagan, J. L. Namy, Tetrahedron 1986, 42, 6573; b) G. A. Molander, Chem. Rev. 1992, 92, 29; c) G. A. Molander, Org. React. 1994, 46, 211; d) G. A. Molander, C. R. Harris, Chem. Rev. 1996, 96, 307; e) T. Skrydstrup, Angew. Chem., Int Ed. Engl. 1997, 36, 345; f) G. A. Molander, C. R. Harris, Tetrahedron 1998, 54, 3321; g) A. Krief, A. M. Laval, Chem. Rev. 1999, 99, 745; h) P. G. Steel, J. Chem. Soc., Perkin Trans. 1 2001, 2727; i) H. B. Kagan, Tetrahedron 2003, 59, 10351; j) J. M. Concellón, H. RodríguezSolla, Chem. Soc. Rev. 2004, 33, 599; k) M. Berndt, S. Gross, A. Hölemann, H. U. Reissig, Synlett 2004, 422; 1) K. Gopalaiah, H. B. Kagan, New J. Chem. 2008, 32, 607; m) J. M. Concellón, H. Rodríguez-Solla, C. Concellón, V. del Amo, Chem. Soc. Rev. 2010, 39, 4103; n) C. Beemelmanns, H. U. Reissig, Chem. Soc. Rev. 2011, 40, 2199; m) H. Y. Harb, D. J. Procter, Synlett 2012, 23, 6.

[4] For a review on the chemistry of $\mathrm{SmI}_{2}-\mathrm{H}_{2} \mathrm{O}$, see: M. Szostak, M. Spain, D. Parmar, D. J. Procter, Chem. Commun. 2012, 48, 330.

[5] For reviews on the use of $\mathrm{SmI}_{2}$ in natural product synthesis, see: a) D. J. Edmonds, D. Johnston, D. J. Procter, Chem. Rev. 2004, 104, 3371; b) K. C. Nicolaou, S. P. Ellery, J. S. Chen, Angew. Chem., Int. Ed. 2009, 48, 7140; c) M. Szostak, D. J. Procter, Angew. Chem., Int. Ed. 2011, 50, 7737 .

[6] For reviews on the influence of additives on properties of $\mathrm{SmI}_{2}$, see: a) H. B. Kagan, J. L. Namy, in 'Lanthanides: Chemistry and Use in Organic Synthesis', Ed. S. Kobayashi, Springer, New York, 1999, p. 155; b) A. Dahlén, G. Hilmersson, Eur. J. Inorg. Chem. 2004, 3393; c) R. A. Flowers, II, Synlett 2008, 1427.

[7] E. Hasegawa, D. P. Curran, J. Org. Chem. 1993, 58,5008 .
[8] P. R. Chopade, E. Prasad, R. A. Flowers, II, $J$. Am. Chem. Soc. 2004, 126, 44.

[9] E. Prasad, R. A. Flowers, II, J. Am. Chem. Soc. 2005, 127, 18093 .

[10] a) A. Yacovan, I. Bilkis, S. Hoz, J. Am. Chem. Soc. 1996, 118, 261; b) A. Tarnopolsky, S. Hoz, Org. Biomol. Chem. 2007, 5, 3801; c) A. Tarnopolsky, S. Hoz, J. Am. Chem. Soc. 2007, 129, 3402; d) M. Amiel-Levy, S. Hoz, J. Am. Chem. Soc. 2009, 131, 8280; e) S. K. Upadhyay, S. Hoz, J. Org. Chem. 2011, 76, 1355.

[11] D. V. Sadasivam, J. A. Teprovich, Jr., D. J. Procter, R. A. Flowers, II, Org. Lett. 2010, 12, 4140.

[12] a) T. K. Hutton, K. W. Muir, D. J. Procter, Org. Lett. 2003, 5, 4811. ; b) G. Guazzelli, L. A. Duffy, D. J. Procter, Org. Lett. 2008, 10, 4291.

[13] L. A. Duffy, H. Matsubara, D. J. Procter, J. Am. Chem. Soc. 2008, 130, 1136.

[14] D. Parmar, L. A. Duffy, D. V. Sadasivam, H Matsubara, P. A. Bradley, R. A. Flowers, II, D. J. Procter, J. Am. Chem. Soc. 2009, 131, 15467.

[15] a) K. D. Collins, H. Matsubara, M. Spain, D. J. Procter, J. Am. Chem. Soc. 2009, 131, 7214; b) M. Szostak, M. Spain, D. J. Procter, Nat. Prot. 2012, 7, 970.

[16] D. Parmar, K. Price, M. Spain, H. Matsubara, P. A. Bradley, D. J. Procter, J. Am. Chem. Soc. 2011, 133, 2418.

[17] K. D. Collins, J. M. Oliveira, G. Guazzelli, B. Sautier, S. De Grazia, H. Matsubara, M. Helliwell, D. J. Procter, Chem. Eur. J. 2010, 16, 10240 .

[18] B. Sautier, S. E. Lyons, M. R. Webb, D. J. Procter, Org. Lett. 2012, 14, 146.

[19] T. M. Baker, L. A. Sloan, L. H. Choudhury, M. Murai, D. J. Procter, Tetrahedron: Asymmetry 2010, 21, 1246.

[20] M. D. Helm, M. Da Silva, D. Sucunza, M. Helliwell, D. J. Procter, Tetrahedron 2009, 65, 10816.

[21] M. Szostak, M. Spain, D. J. Procter, Chem. Commun. 2011, 47, 10254.

[22] M. Szostak, K. D. Collins, N. J. Fazakerley, M. Spain, D. J. Procter, Org. Biomol. Chem. 2012 DOI: $10.1039 / \mathrm{C} 2 \mathrm{OB} 00017 \mathrm{~B}$.

[23] M. Szostak, M. Spain, D. J. Procter, Org. Lett. 2012, 14,840 . 